\title{
Permittivitätsmessung mit open-ended koaxialen und koplanaren Tastköpfen zur schnellen Quantifizierung von Ödemen im Hirngewebe
}

\author{
$\underline{\text { T Reinecke }^{+}, \text {L Hagemeier }}$, S Ahrens ${ }^{+}$, M Klintschar ${ }^{*}$, S Zimmermann ${ }^{+}$
}

\begin{abstract}
+Institut für Grundlagen der Elektrotechnik und Messtechnik, Fachgebiet Sensorik und Messtechnik, Leibniz Universität Hannover, Appelstraße 9a, 30167 Hannover, Tel.: +49 511 762-4228, www.geml.uni-hannover.de *Institut für Rechtsmedizin, Medizinische Hochschule Hannover
\end{abstract}

\section{Kurzfassung}

In einer Autopsie kann die Quantifizierung der Ödeme im Hirngenwebe Hinweise auf die Todesursache liefern. Dies ist insbesondere im Falle multipler Erkrankungen von großer Wichtigkeit, oder wenn keine offensichtlichen Anzeichen für die Todesursache vorzufinden sind, wie zum Beispiel bei der Unterscheidung zwischen plötzlichem Kindstod und dem Tod durch ein Schütteltrauma. Bislang existiert kein standardisiertes Verfahren zur Quantifizierung von Ödemen im menschlichen Gehirn. Die gängige Praxis ist die Bestimmung des Gehirngewichts sowie die subjektive Beurteilung der Ventrikelkompression und Schwellungen im Gehirn, was fehleranfällig ist. Deswegen ist das Ziel unserer Arbeit ein schnelles, präzises und kostengünstiges Verfahren für die objektive Quantifizierung des Ödemgehalts zu entwickeln. Hierzu wird der quantitative Zusammenhang zwischen Feuchtegehalt und Permittivität von Hirngewebe ausgenutzt. In einem ersten Schritt konnten wir mittels Messungen unter Verwendung einer koaxialen Messkammer zeigen, dass zwischen der Gewebefeuchte und der Permittivität ein linearer Zusammenhang besteht. Dieses Verfahren ist sehr exakt, hat aber den Nachteil eines erhöhten Präparationsaufwands da für jede Messung die Gewebeprobe in die Messkammer eingebracht werden muss. Deswegen ist eine sequentielle Suche nach Ödemen im Gewebe sehr zeitaufwändig. Um eine Probenpräparation komplett zu umgehen, werden ein open-ended koaxialer und ein koplanarer 
Tastkopf für die Bestimmung der Permittivität entwickelt und in Bezug auf die erreichbare Messgenauigkeit miteinander verglichen.

\section{Einleitung}

Die Physiologie des Menschen gestattet nur geringe Toleranzen der Gewebsfeuchte. Stärkere Abweichungen von der Norm deuten daher auf schwerwiegende Erkrankungen hin. Wenngleich das Vorliegen einer Erkrankung bei einer möglicherweise unbekannten Leiche nicht ausgeschlossen werden kann, so stellen insbesondere in Bezug auf das Hirnödem - Wassereinlagerungen im Sinne eines Ödems hier am Ehesten pathologische Prozesse im Rahmen der Agonie dar. Der Zusammenhang zwischen Wassereinlagerungen im Gehirn und der Dauer der Agonie wurden zuerst in [1] untersucht. Hierin ist dargelegt, dass eine lange Agoniezeit mit einer starken Ödembildung im Gehirn einhergeht. Dies ist darin begründet, dass während der Agonie das Gehirn großem Stress ausgesetzt ist, es deswegen expandiert und sich Ödeme bilden. Bei einer kurzen Agoniezeit hingegen werden alle Körperfunktionen sofort gestoppt, dem Gehirn also keine Zeit zur Expansion gegeben. Deswegen sind in diesem Fall auch keine Ödeme vorzufinden. Agoniezeit und Wassergehalt im Gehirn stehen also in direkter Verbindung zueinander. Daher kann die Messung des Wassergehalts zur richtigen Diagnose führen, wenn multiple mögliche Todesursachen vorliegen, oder wenn keine offensichtlichen Ursachen für das Versterben aufzufinden sind, wie zum Beispiel bei der Unterscheidung zwischen plötzlichem Kindstod und dem Tod durch ein Schütteltrauma. Bis heute existiert allerdings in der Rechtsmedizin kein standardisiertes Verfahren zur Quantifizierung des Ödemgehalts. Die Beurteilung erfolgt subjektiv über die Begutachtung von Schwellungen und der Ventrikelkompression sowie über die Bestimmung des Gehirngewichts und ist daher anfällig für Fehler. Ein Verfahren für die objektive, exakte Bestimmung des Wassergehalts im Gewebe ist die Thermogravimetrie. Hierbei wird eine Gewebeprobe entnommen, gewogen, getrocknet und anschließend erneut gewogen. Aus der Massendifferenz vor und nach der Trocknung kann dann der Wassergehalt bestimmt werden. Die Trocknung erfolgt entweder in Trockenschränken, was mehrere Tage in Anspruch nimmt, 
oder mittels Mikrowellen [2], was mit einem erhöhten apparativen Aufwand und deswegen mit erhöhten Kosten verbunden ist.

Ziel unserer Arbeit ist es daher, ein kostengünstiges Verfahren zum einfachen Einsatz in einer Autopsie zu entwickeln, was die schnelle und exakte Quantifizierung von Ödemen im menschlichen Gehirn erlaubt. Dazu wird der quantitative Zusammenhang zwischen der relativen Permittivität und dem Wassergehalt ausgenutzt [3]. In einem ersten Schritt wurden dazu Transmissionsmessungen mit einer koaxialen Messkammer durchgeführt [4]. Hierbei zeigte sich, dass ein linearer Zusammenhang zwischen Gewebefeuchte und dem Realteil der relativen Permittivität besteht und dass eine exakte Bestimmung der Gewebefeuchte mit einem Fehler von unter $2 \%$ über eine Permittivitätsmessung möglich ist. Nachteilig an diesem Verfahren ist, dass die Gewebeprobe in die koaxiale Messkammer eingebracht werden muss, wodurch sich die Messzeiten erhöhen und damit eine sequentielle Messung an mehreren Stellen im Gehirn sehr zeitaufwendig ist. Ein weit verbreitetes Verfahren zur zerstörungsfreien Bestimmung der Permittivität ist die Verwendung eines open-ended koaxialen Tastkopfs. Dieser wird einfach auf die Gewebeprobe gepresst und die Permittivität wird mittels einer Reflexionsmessung bestimmt. In einer vorangegangenen Arbeit zeigte sich hierbei, dass die Reflexionsmessung gute Ergebnisse erzielt. Die Bestimmung des Wassergehalts im Hirngewebe ist mit einem maximalen Fehler von 3\% möglich, allerdings ist dieses Verfahren ungenauer als die Transmissionsmessung mit der koaxialen Messammer. Deswegen wird im Rahmen dieser Arbeit die Bestimmung des Wassergehalts im menschlichen Gehirn über eine Permittivitätsmessung unter Verwendung einer Koplanarleitung untersucht. Dieses Verfahren verbindet die Vorteiler beider eben genannten Verfahren, da mit der Koplanarleitung Transmissionsmessungen durchgeführt werden können, sie aber zusätzlich als Tastkopf ausgeführt werden kann und somit keine Probenentnahme notwendig ist. 


\section{Theorie}

In diesem Abschnitt werden die theoretischen Grundlagen für die Bestimmung der relativen Permittivität einer Gewebeprobe dargestellt. Hierbei wird angenommen, dass es sich bei der Wellenausbreitung auf der Koplanarleitung um eine sogenannte transversalelektromagnetische (TEM) Welle handelt.

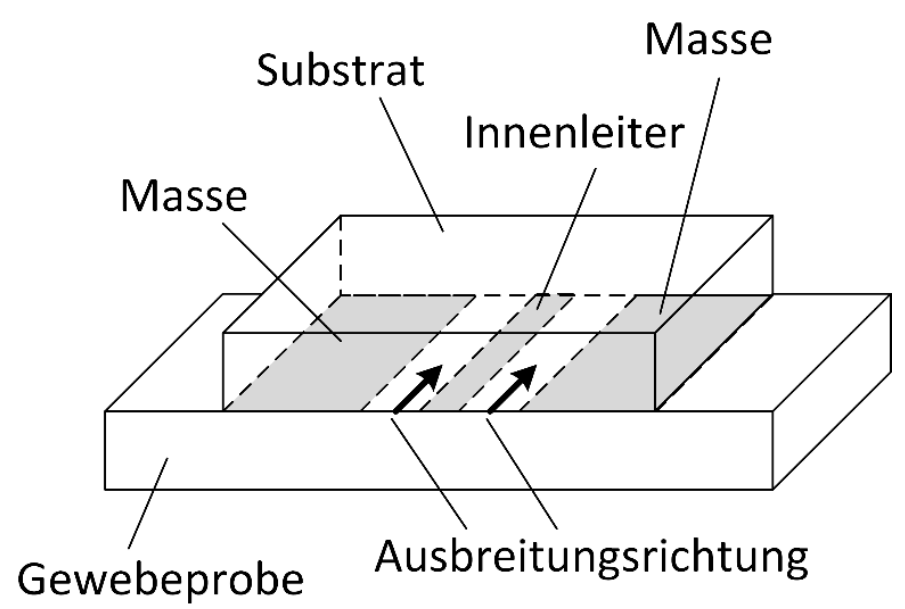

Abbildung 1: Schematische Darstellung einer Koplanarleitung

In Abb. 1 ist schematisch eine Koplanarleitung dargestellt. Sie besteht aus einem Innenleiter und dazu beidseitig jeweils einer Massefläche. Die Leiterstruktur befindet sich auf einem Substrat. Die Wellenausbreitung in einer Koplanarleitung erfolgt längs des Innenleiters. Zur Bestimmung der Permittivität wird die Leiterstruktur einfach auf die Gewebeprobe gepresst und eine Transmissionsmessung durchgeführt. In Abb. 2 sind die Ergebnisse einer Simulation mit CST Microwave Studio 2013 dargestellt. Das Substrat ist Fr4 mit einer relativen Permittivität von $\varepsilon_{\mathrm{r}}=4,3$, welches ebenfalls für den Aufbau des Taskopfs verwendet wird. Die Breite des Innenleiters, sowie die Abstände zwischen Innenleiter und Massefläche sind auf einen Wellenwiderstand $Z_{0}=50 \Omega$ bei unbelasteter Koplanarleitung ausgelegt. Aus den Simulationsergebnissen ist zu erkennen, dass sich sowohl Feldanteile im Substrat, als auch innerhalb der Gewebeprobe befinden, weswegen die Permittivität der Probe die Wellenausbreitungseigenschaften der Koplanarleitung beeinflusst. Damit weicht der Wellenwiderstand $\mathrm{Z}_{1}$ der auf eine Gewebeprobe gepressten Koplanarleitung von dem der unbelasteten Leitung ab. 


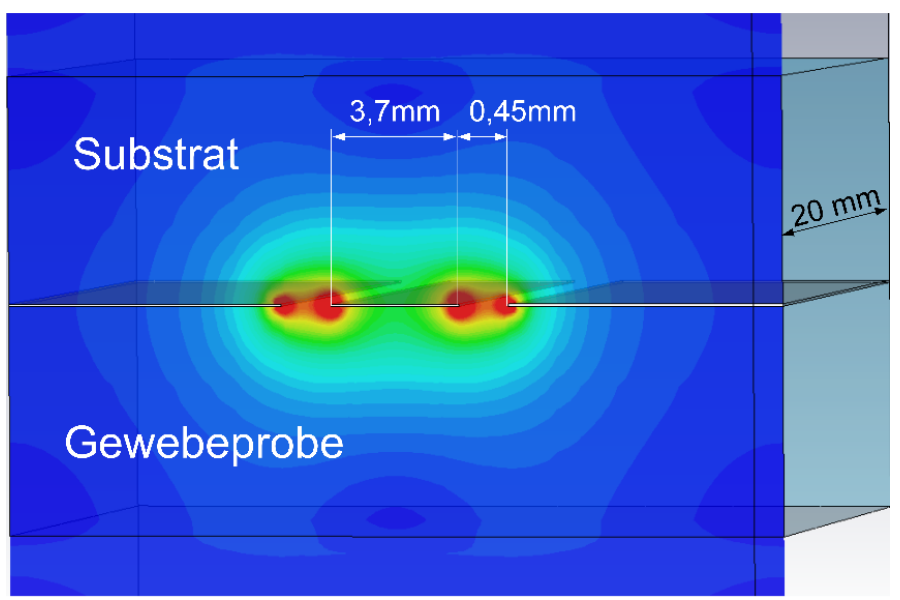

Abbildung 2: $\quad$ CST Simulation einer Koplanarleitung

Die Gewebeprobe ist nicht magnetisch, daher gilt für die relative Permeabilität $\mu_{\mathrm{r}}=1$. Damit kann der Transmissionskoeffizient einer TEM Welle in einer Koplanarleitung beschrieben werden als

$$
z=e^{-j \cdot \frac{\omega}{c_{0}} \cdot \sqrt{\varepsilon_{r, e f f}} \cdot l} .
$$

Hierbei ist $\omega$ die Kreisfrequenz, $c_{0}$ die Lichtgeschwindigkeit, $l$ die Länge der Koplanarleitung und $\varepsilon_{r, \text { eff }}=\varepsilon_{r, \text { eff }}{ }^{\prime}+\mathrm{j} \varepsilon_{r, \text { eff }}$ " die komplexe effektive Permittivität. Der Realteil $\varepsilon_{r, \text { eff }}$ ' ist ein Maß für die Polarisierbarkeit und der Imaginärteil $\varepsilon_{r, \text { eff }}$ " ein Maß für die dielektrischen Verluste. Die effektive Permittivität setzt sich aus einer Überlagerung der Permittivität des Substrats und der Permittivität des Materials unterhalb des Tastkopfes zusammen. Die effektive Permittivität des unbelasteten Tastkopfes wurde sowohl aus einer Simulation, als auch experimentell zu $\varepsilon_{r, e f f}(L u f t)=2,6$ bestimmt. Damit kann der Reflexionsfaktor der Koplanarleitung bestimmt werden zu

$$
\Gamma=\frac{Z_{1}-Z_{0}}{Z_{1}+Z_{0}}=\frac{\sqrt{\frac{1}{\varepsilon_{r, e f f(\text { Gewebe })}}}-\sqrt{\frac{1}{\varepsilon_{r, e f f(\text { Luft })}}}}{\sqrt{\frac{1}{\varepsilon_{r, \text { eff }}(\text { Gewebe })}}+\sqrt{\frac{1}{\varepsilon_{r, \text { eff }}(\text { Luft })}}} .
$$

Hierbei ist $\varepsilon_{r, \text { eff }}$ (Gewebe) die effektive Permittivität des Tastkopfs, wenn dieser auf eine Gewebeprobe gepresst wird und stellt im Rahmen dieser Arbeit die Messgröße als Maß für den Wassergehalt im Gewebe dar. Nach [5] kann mit $\Gamma$ und $z$ der Streuparameter $S_{21}$, welcher die Transmission einer TEM Welle beschreibt als 


$$
S_{21}=\frac{b_{2}}{a_{1}}=\frac{z \cdot\left(1-\Gamma^{2}\right)}{1-z^{2} \Gamma^{2}}
$$

ausgedrückt werden. (3) stellt damit ein Gleichungssystem mit $\varepsilon_{r, \text { eff }}$ (Gewebe) und $\varepsilon_{r, \text { rff }}$ "(Gewebe) als Unbekannten dar. Der Streuparameter $\mathrm{S}_{21}$ kann mit einem Netzwerkanalysator gemessen werden. Wir verwenden dafür den low-cost Netzwerkanalysator $V W N A 3$ von SDR-Kits bei einer Frequenz von 500 Mhz. Nach [6] kann die Lösung des Gleichungssystems durch umstellen von (3) zu

$$
\frac{z \cdot\left(1-\Gamma^{2}\right)}{1-z^{2} \Gamma^{2}}-S_{21}=0
$$

nummerisch erfolgen. Dafür verwenden wird der Newton Algorithmus verwendet.

\section{Messungen}

In diesem Abschnitt werden Messungen der effektiven Permittivität mit dem entwickelten koplanaren Tastkopf an menschlichem Hirngewebe vorgestellt. Der Wassergehalt in der jeweils gemessenen Probe wird mit einem kommerziellen Feuchteanalysator, dem Smart System 5 von CEM durchgeführt. Dieser ist sehr exakt, mit einer Standardabweichung von $\sigma=0.1 \%$ und dient als Referenz. Im Vorfeld zu den Messungen muss einmalig der Messaufbau kalibriert werden. Hierzu wird eine sogenannte $\mathrm{T}$ (hrough)O(pen)S(hort)M(atch) Kalibrierung verwendet, bei der definierte Lasten anstelle des Tastkopfes angeschlossen werden und so Störeinflüsse durch Kabel und Übergänge aus den Messergebnissen eliminiert werden. 


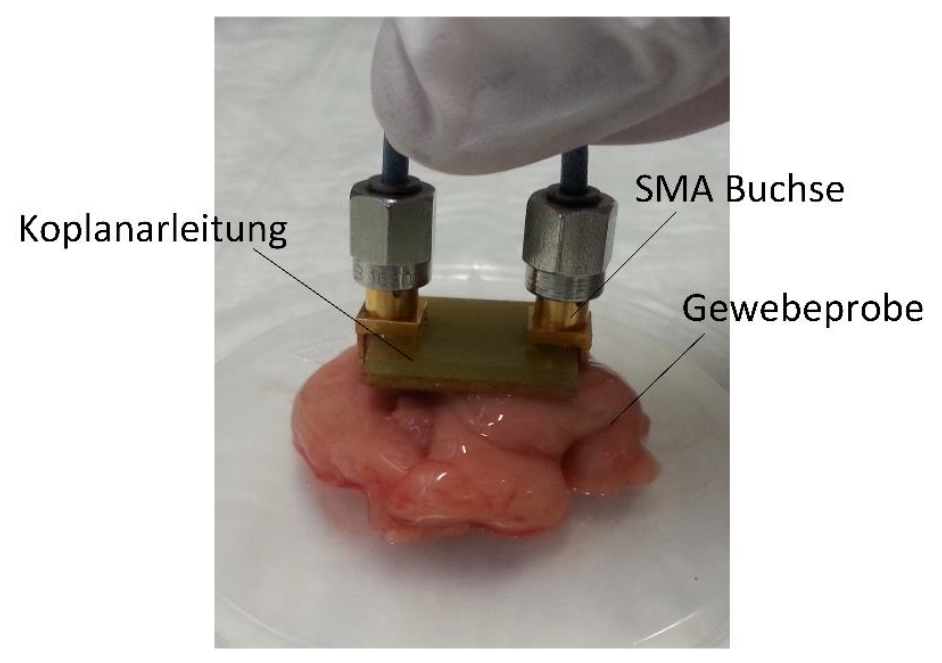

Abbildung 3: $\quad$ Messung einer menschlichen Hirngewebeprobe mit dem koplanaren Tastkopf.

In Abb. 3 ist eine Permittivitätsmessung mit dem entwickelten Tastkopf dargestellt dieser wird einfach von Hand auf die Gewebeprobe gepresst. Dann wird mit dem Netzwerkanalysator eine Messung des $S_{21}$ durchgeführt und mit dem beschriebenen Algorithmus die effektive Permittivität berechnet. Dieser Vorgang dauert etwa 20s pro Gewebeprobe und ist damit deutlich schneller als der kommerzielle Feuchteanalysator welcher Messzeiten von bis zu 10min für eine Probe benötigt.

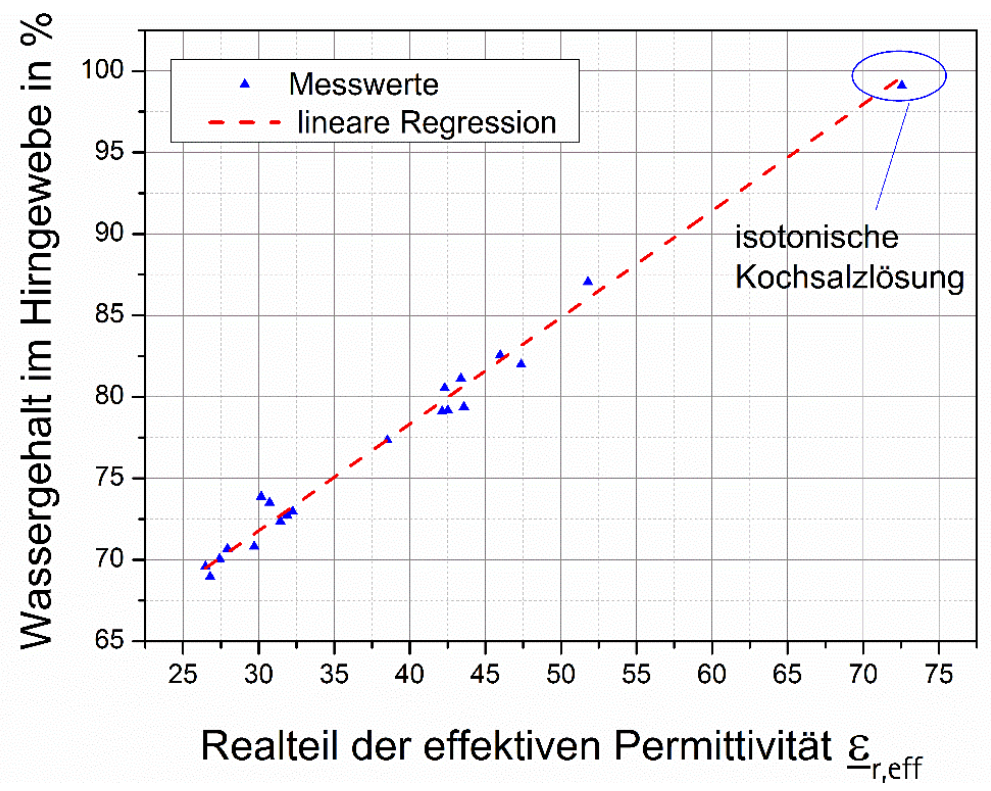

Abbildung 4: $\quad$ Lineare Regression zwischen dem Wassergehalt im Hirngewebe, gemessen mit dem Smart System 5, und dem Realteil der effektiven Permittivität, gemessen mit dem koplanaren Tastkopf. 
In Abb. 4 ist der lineare Zusammenhang zwischen dem Wassergehalt im Hirngewebe und dem Realteil der effektiven Permittivität dargestellt. Der Wert bei 99,1\% Feuchte wurde aus einer Messung von isotonischer Kochsalzlösung ermittelt, da diese eine ähnliche Elektrolytzusammensetzung wie das Serum im Gehirn hat [7]. Dieser Wert stellt den theoretischen maximalen Wassergehalt dar. Eine statistische Analyse liefert einen maximalen Fehler von 2\% für die Bestimmung des Wassergehalts mittels einer Permittivitätsmessung mit koplanaren Tastkopf.

\section{Zusammenfassung}

In dieser Arbeit präsentieren wir ein kostengünstiges Messsystem zur schnellen und exakten Bestimmung des Wassergehalts im menschlichen Hirngewebe. Hierbei wird der lineare Zusammenhang zwischen der relativen Permittivität des Gewebes und dem Wassergehalt ausgenutzt. Die Messung erfolgt mit einem neu entwickelten koplanaren Tastkopf. Hiermit ist die Bestimmung des Feuchtegehalts mit einem maximalen Fehler von 2\% möglich, womit das Verfahren ebenso exakt ist wie Messungen mit einer koaxialen Messkammer aus einer vorangegangenen Arbeit. Vergleichsmessungen mit einem open-ended koaxialen Tastkopf liefern einen Fehler von 3\% für die Bestimmung des Wassergehalts im Gewebe. Ein weiterer Vorteil des koplanaren Tastkopfes gegenüber dem open-ended koaxialen Tastkopf ist die deutlich einfachere Kalibrierung mit TOSM Kalibrierstandards, während bei der Verwendung eines open-ended koaxialen Tastkopfes Referenzmaterialien mit definierter Permittivität zur Kalibrierung vermessen werden müssen.

\section{Literatur}

[1] Bogomolov DV, Dolzhanskiĩ OV, Kalashnikov DP, Luzin AV (2006), "Quantitative assessment of brain hydration in determination of death speed and type of tanatogenesis in forensic medical practice", Sud Med Ekspert. 49(1):14-6

[2] Collins Michael J., "Analytical method for determining volatiles, solids and solvent extractables", US4753889 A, 1988 
[3] A. Kraszewski, Microwave Aquametry-Electromagnetic Wave Interaction with Water-Containing Materials. New York: IEEE Press, 1996

[4] Reinecke, T.; Hagemeier, L.; Schulte, V.; Klintschar, M.; Zimmermann, S., "Quantification of edema in human brain tissue by determination of electromagnetic parameters," SENSORS, 2013 IEEE, pp.1390,1393, 3-6 Nov. 2013

[5] Nicolson, A.M.; Ross, G. F., "Measurement of the Intrinsic Properties of Materials by Time-Domain Techniques," Instrumentation and Measurement, IEEE Transactions on , vol.19, no.4, pp.377,382, 1970

[6] Baker-Jarvis, J.; Vanzura, E.J.; Kissick, W.A., "Improved technique for determining complex permittivity with the transmission/reflection method," Microwave Theory and Techniques, IEEE Transactions on , vol.38, no.8, pp.1096,1103, 1990

[7] Schmidt R. F., Thews G., "Human Physiology", Springer,1989 